\title{
Movimento de gaveta em joelhos de cães submetidos à estabilização extracapsular após secção do ligamento cruzado cranial in vitro
}

\author{
Drawer movement in canine knees undergoing extracapsular stabilization after cranial cruciate \\ ligament rupture in vitro
}

\begin{abstract}
Bernardo Kemper Silvia Manduca Trapp $^{\mathrm{II}}$ Tássio Falleiros Porto ${ }^{\mathrm{III}}$ Flavio Antonio Barca ${ }^{\mathrm{II}}$
\end{abstract}
\section{RESUMO}

A técnica TightRope, que procura aperfeiçoar a estabilização extra-capsular com sutura lateral, por meio da realização de mínimas incisões e criação de túneis ósseos em pontos isométricos, que permitem a inserção de mecanismo que anula o movimento de gaveta, além de reduzir a ocorrência de complicações graves. Dessa forma, objetivou-se avaliar a modificação da técnica TightRope em joelhos de cadáveres caninos com a finalidade de disponibilizar um procedimento simples e de custo reduzido para tratamento da Ruptura do ligamento cruzado cranial (RLCCr). Experimentalmente, foram utilizados 20 membros pélvicos de dez cadáveres caninos, provenientes do setor de patologia da Universidade Norte do Paraná, os quais pesavam entre 6,3 e 24kg. Para estabilização do $L C C r$, rompido intencionalmente, foi adotada a técnica de TightRope modificada utilizando fio de poliamida, cavilha $e$ emprego de um parafuso ortopédico para proporcionar a fixação óssea. Comparando os valores de deslocamento obtidos durante a realização do movimento de gaveta previamente à ruptura do LCCr e após a realização do procedimento cirúrgico, observou-se que a modificação da técnica TightRope promoveu estabilidade significativa para a maioria (12 de 20) dos joelhos testados $(P=0,0033)$. Contudo, essa estabilidade foi inferior, quando comparada à estabilidade do ligamento intacto.

Palavras-chave: articulação, ponto isométrico, tratamento cirúrgico.

\section{ABSTRACT}

The Tightrope technique, that has been developed, aims to improve the extra-capsular stabilization with lateral suture through smaller incisions and creating isometric bone tunnels at points that allow the inclusion of a mechanism that overrides the movement of drawer and reduce the occurrence of serious complications. Therefore, the objective was to evaluate the Tightrope modified technique in canine cadaver knees in order to provide a simple and cost effective treatment for cranial cruciate ligament rupture (CCrLR). Experimentally; we used 20 pelvic limbs of ten canine corpses from the pathology service at the University of Northern Parana, which weighed between 6.3 and $24 \mathrm{~kg}$. To stabilize the cranial cruciate ligament (CCrL), which has been broken intentionally, the Tightrope modified technique was adopted by using modified polyamide yarn, pin and a grub screw to provide orthopedic bone fixation. Comparing the displacement values obtained during the movement of the drawer before the break of CCrL and after the surgical procedure was observed that the Tightrope modified technique provided significant stability for the majority (12 of 20) of the knees tested $(P=0.0033)$. However, the stability of the technique was lower than the stability of the intact ligament.

Key words: joint, isometric point, surgical technique.

\section{INTRODUÇÃO}

O joelho é formado por uma complexa articulação femur-tíbia-patelar (AFTP), composta por duas articulações funcionalmente distintas: a fêmur-tibial, que suporta a maior parte do peso, e a fêmur-patelar, que tem como funções manter a patela no sulco troclear, estabilizando o movimento de extensão e aumentando a eficácia mecânica do grupo de músculos quadrícipes (VASSEUR, 2003; CONSTANTINESCU, 2005).

A estabilidade da AFTP é proporcionada pelos ligamentos colateral medial, colateral lateral, cruzado cranial e cruzado caudal, sendo que o nome dos dois últimos se deve ao local de inserção no

${ }^{\mathrm{I}}$ Faculdade de Medicina Veterinária e Zootecnia (FMVZ), Universidade Estadual Paulista (UNESP), Campus de Botucatu Distrito de Rubião Junior, 18618-970, Botucatu, SP, Brasil. E-mail:bkemper@bol.com.br. Autor para correspondência.

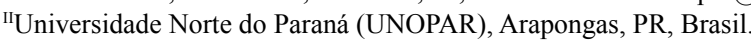

"IIMedico Veterinário Autônomo, Apucarana, PR, Brasil. 
platô tibial. Secundariamente, o ligamento cruzado cranial (LCCr) também é importante na limitação de angulações varus e valgus (ROOSTER et al., 2006).

O LCCr é dividido em dois componentes funcionais, a faixa craniomedial e a faixa caudolateral, as quais se inserem em diferentes pontos no platô tibial. A faixa craniomedial permanece estirada em todas as fases de extensão e flexão, enquanto que a faixa caudolateral permanece estirada em extensão e frouxa em flexão (PIERMATTEI, et al., 2006; SCHULZ, 2007).

A RLCCr, que pode ser completa ou parcial, é considerada a enfermidade ortopédica mais importante na espécie canina, sendo a causa mais comum de claudicação do joelho e um dos principais fatores etiológicos da doença articular degenerativa da AFTP (GRIFFON, 2010). A RLCCr em cães é uma doença multifatorial envolvendo fatores genéticos, conformacionais e inflamatórios que, em conjunto, criam um desequilíbrio entre a força biomecânica aplicada no ligamento e sua habilidade em sustentar essa carga, levando, eventualmente, à ruptura e a instabilidade articular (GRIFFON, 2010; COMERFORD et al., 2011).

O teste movimento de gaveta é a translação cranial da tíbia em relação ao fêmur, que possibilita o diagnóstico clínico para RLCCr (PALMER, 2005). O movimento de gaveta deve ser realizado com o membro pélvico flexionado e também em extensão (VASSEUR, 2003). A sensibilidade do teste aumenta consideravelmente quando realizado sob anestesia, reduzindo o risco de resultados falsonegativos (CAROBBI \& NESS, 2009). De acordo com MOORE \& READ (1996), para que o teste seja considerado positivo, o deslocamento crânio-caudal da tíbia em relação ao fêmur deve ser superior a $2 \mathrm{~mm}$. A demonstração do deslocamento cranial da tíbia em relação ao fêmur por meio da realização do exame radiográfico, concomitante ao teste de compressão tibial, é uma opção para a confirmação da RLCCr (PALMER, 2005).

Existem várias formas de tratar a RLCCr, entretanto, a escolha terapêutica é influenciada pela idade, tamanho corporal e função do animal, além das considerações econômicas do proprietário (VASSEUR, 2003). O tratamento clínico conservador consiste basicamente em restrição da atividade física, controle alimentar para redução de peso corporal, analgesia, fisioterapia e, eventualmente, aplicação de bandagem em cães até $10 \mathrm{~kg}$ de peso corporal (PIERMATTEI, et al., 2006; COMERFORD, 2007). Evolução dos sinais clínicos e osteoartrose gradativa são observados em cães entre 15 e $20 \mathrm{~kg}$, submetidos ao tratamento conservador (SCHULTZ, 2007). De acordo com KIM et al. (2008), a estabilização cirúrgica deve ser recomendada a todos os pacientes, principalmente aos cães com peso corporal superior a 10 e $15 \mathrm{~kg}$.

As técnicas cirúrgicas que estabilizam a articulação do joelho são divididas em três grupos principais: método extracapsular, método intracapsular e osteotomias proximais à tíbia (PIERMATTEI, et al., 2006; KIM et al., 2008). Contudo, nenhuma técnica é considerada de eleição (CONZEMIUS et al., 2005; LAZAR et al., 2005). Outra opção cirúrgica são as próteses para substituição total do joelho de cães com doença articular degenerativa (DAD), cujos resultados de sua aplicação têm demonstrado viabilidade para cães com peso corporal entre $12 \mathrm{e}$ $60 \mathrm{~kg}$ (LISKA \& DOYLE, 2009).

As técnicas extra-capsulares requerem menos instrumentos especializados, são geralmente mais simples e rápidas, apresentam melhor custobenefício e são mais acessíveis (JERRAM \& WALKER, 2003). Atualmente, um dos métodos mais utilizados é a sutura fabelo-tibial lateral (SFTL), independentemente do tamanho do animal, o fio, geralmente, é passado com agulha curva ao redor do ligamento fabelo-femoral e por um orifício criado na crista tibial. Em substituição à fixação na fabela, pode ser empregada uma âncora no côndilo lateral (SCHULZ, 2007). Procedimentos que requerem a SFTL são tecnicamente menos complexos do que métodos intra-articulares ou modificadores da biomecânica (osteotomias) e, por isso, continuam a ser praticados por muitos cirurgiões. A melhor sutura lateral é aquela que elimina a translação crânio-caudal da tíbia, cuja colocação não interfira na distância entre os pontos de ancoragem durante os movimentos de flexão e extensão (HULSE et al., 2010). Contudo, independentemente do ponto exato de fixação da sutura lateral, mudanças substanciais na tensão desta podem ocorrer durante o movimento da articulação. Essa mudança na tensão aumenta significativamente com a flexão da articulação (FISCHER, et al., 2010; TONKS et al., 2010).

Em Medicina Veterinária, para tratamento da RLCCr em cães, foi recentemente descrita a adaptação da técnica de estabilização da articulação tíbio-társica aplicada em pessoas. Denominada TightRope, essa técnica minimamente invasiva, através de túneis ósseos, permite a inserção de um mecanismo extracapsular para estabilização do LCCr. Esse procedimento anula o movimento de gaveta e reduz a ocorrência de complicações graves. A intenção desse método é aperfeiçoar a técnica de sutura lateral,

Ciência Rural, v.43, n.6, jun, 2013. 
colocando em pontos isométricos um implante de maior resistência e rigidez com fixação óssea em tíbia e fêmur, especificamente para reparação da RLCCr (Tight Rope $\mathrm{CCl}^{\circledR}$, Arthrex Vet Systems, Naples, FL, USA). Devido à necessidade de suportar a criação de túneis ósseos, a técnica é indicada para animais com peso corpóreo superior ou igual a $15 \mathrm{~kg}$. A aplicação dessa técnica em cães com deficiência do LCCr tem proporcionado bons resultados e, quando comparada com a TPLO, requer menor tempo de anestesia e cirurgia e apresenta menor taxa de complicações pós-operatórias que requerem intervenções (COOK et al., 2010).

O objetivo deste trabalho foi avaliar o efeito da aplicação da técnica TightRope modificada em joelhos de cadáveres caninos, por meio da mensuração do movimento de gaveta em extensão de $135^{\circ}$.

\section{MATERIAL E MÉTODOS}

Experimentalmente foram utilizados 20 membros pélvicos oriundos de dez cadáveres caninos provenientes do Setor de Patologia Animal, os quais pesavam entre 6,3 e $24 \mathrm{~kg}$. Os membros pélvicos incluídos neste estudo foram livres de alterações macroscópicas durante inspeção visual e de anomalias morfológicas pesquisadas em radiografias realizadas em projeção médio-laterais. Após a seleção dos membros pélvicos, eles foram imediatamente congelados a $-20^{\circ} \mathrm{C}$ e descongelados a temperatura ambiente, 24 horas antes da realização dos procedimentos.

As amostras foram divididas em três grupos: grupo I - antes da ruptura do LCCr; grupo II LCCr rompido; grupo III - após correção da RLCCr pela técnica TightRope modificada.

Após ampla tricotomia nas regiões medial e lateral, desde a articulação coxofemoral até a articulação do tarso, foi realizada artrotomia lateral para expor a articulação do joelho e do LCCr, conforme descrição de Piermattei e colaboradores (2004). Em todos os joelhos, foram seccionados os ligamentos cruzados craniais com o auxílio de uma lâmina de bisturi no 15 , próximo a sua inserção no platô tibial. Posteriormente à confirmação da secção do LCCr por meio do teste do movimento de gaveta, foi realizada a inspeção do espaço articular.

Para estabilização extra-capsular do LCCr, foi adotado o método de TightRope com modificações. $\mathrm{O}$ procedimento cirúrgico envolveu o uso de fio de poliamida $(0,7 \mathrm{~mm})$, cavilha e o emprego de um parafuso ortopédico como forma de fixação óssea.

Na sequência ao acesso cirúrgico realizado na região parapatelar lateral, dissecção caudal e retração da incisão lateral da fáscia, foi possível expor o segmento caudo-lateral da articulação do joelho. Tendo a fabela lateral e a tuberosidade tibial como pontos anatômicos de referência, criou-se túnel ósseo com auxílio de uma broca ortopédica de $2 \mathrm{~mm}$, na região crânio-proximal ao sulco do tendão extensor digital longo, progredindo pela tíbia, em um ângulo ligeiramente craniodistal e atravessando para o seu lado medial. Com auxílio de uma agulha hipodérmica descartável (40x12) como guia, um fio de poliamida $(0,7 \mathrm{~mm})$ atravessou o orifício criado na tíbia, a partir da região lateral em direção à região medial, onde foi inserida uma cavilha (Cão medica, São Paulo) e, com a finalidade de fixá-la, o fio de poliamida retornou pelo mesmo orifício, porém no sentido oposto.

Após palpação cuidadosa e utilizando a mesma broca, uma perfuração foi realizada $2 \mathrm{~mm}$ cranial e distal a fabela lateral, sendo esta guiada para porção distal do fêmur em direção à face medial, imediatamente caudal ao músculo vasto medial, na altura da borda proximal da patela. Auxiliado por uma agulha hipodérmica descartável (40x12), o fio de poliamida foi conduzido pela perfuração, partindo da face lateral em direção à face medial. Posteriormente, por intermédio de broca ortopédica $(2 \mathrm{~mm})$ e chave apropriada, foi inserido um parafuso ortopédico de $2,7 \mathrm{~mm}$ no côndilo femoral medial localizado por palpação, o parafuso foi mantido $3 \mathrm{~mm}$ sobressalente em todos os membros e, após a colocação do fio, apertado em uma volta. Então, com o membro em posição de extensão de $135^{\circ}$, neste parafuso, foi fixado o fio de poliamida com um nó de cirurgião seguido por quatro nós simples. A confecção dos nós e da tensão aplicada foi realizada pela mesma pessoa, sem auxílio de equipamento de mensuração (Figura 1).

Previamente ao rompimento do LCCr, após o rompimento e depois de realizado o método de TightRope modificado, os membros foram posicionados lateralmente à superfície de uma mesa plana e flexionados em um ângulo de $135^{\circ}$, com o auxílio de um artrogoniômetro. Em seguida, foi realizado o teste do movimento de gaveta cranial da tíbia (deslocamento cranial da tíbia) em relação ao fêmur para verificar o grau de deslocamento. Dessa forma, cada membro testado também foi utilizado como seu próprio controle. Todas as medições foram realizadas pelo mesmo avaliador, auxiliado por um paquímetro. Para isso, em todos os joelhos utilizados, fixou-se um pino (Kirschner 1,0mm) na região proximal e outro na distal da inserção do ligamento colateral lateral, no seu ponto de origem no fêmur e o outro no ponto de inserção na tíbia, sem que dificultasse a colocação e deslocamento do fio, que serviram de referência para 


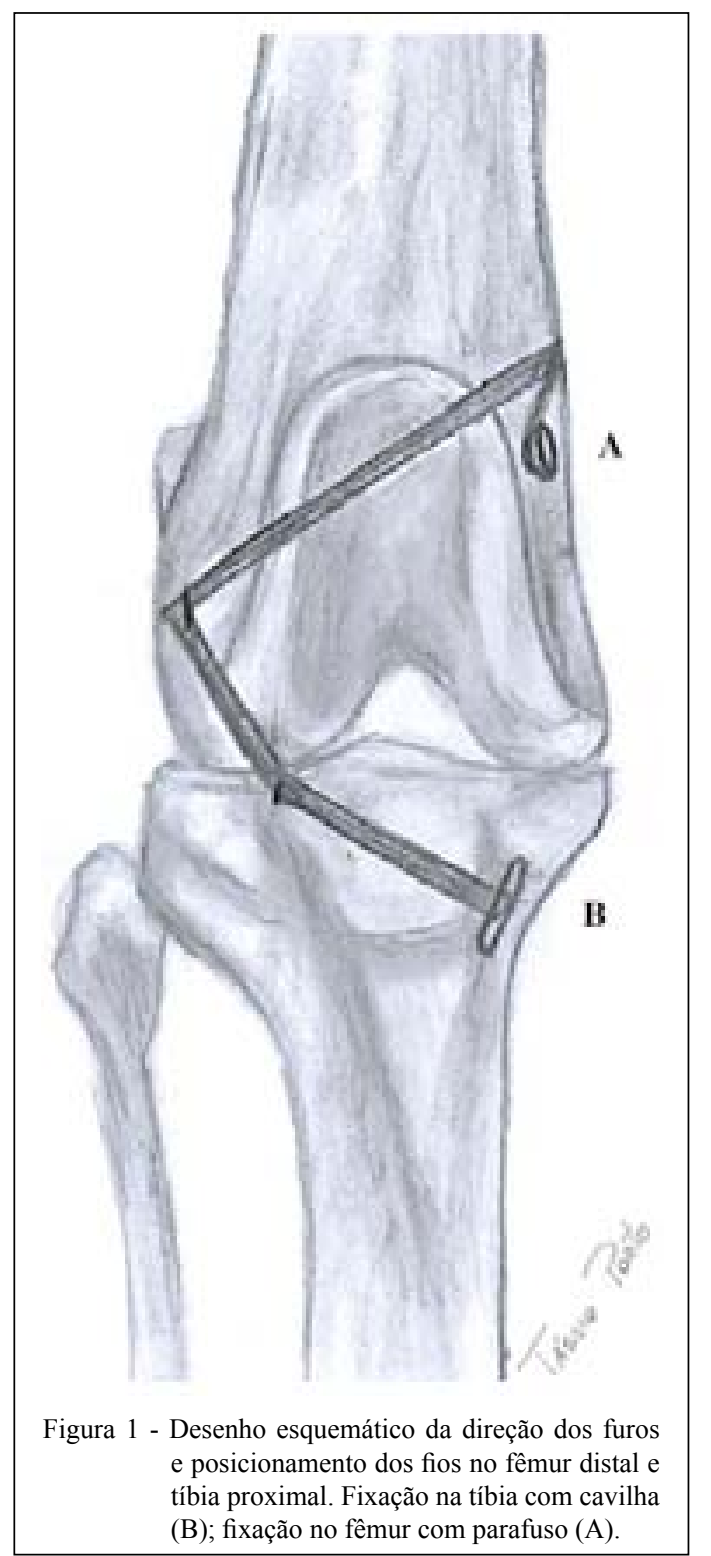

medir o deslocamento da tíbia em relação ao fêmur durante o movimento de gaveta.

Os dados do deslocamento cranial da tíbia foram analisados através de teste não paramétrico de Kruskal-Wallis, devido à falta de normalidade. Para a comparação entre os grupos de movimentação até dois milímetros e acima, foi utilizado o teste exato de Fisher. O nível mínimo de significância para ambos os casos foi de $5 \%$.

\section{RESULTADOS E DISCUSSÃO}

A média dos valores da translação crâniocaudal da tíbia previamente à ruptura do $\mathrm{LCCr}$, após a ruptura e após a realização do procedimento cirúrgico TightRope modificado, foram $0,50 \mathrm{~mm}, 10,70 \mathrm{~mm}$ e $2,25 \mathrm{~mm}$, respectivamente $(\mathrm{P}=0,0033)$ (Tabela 1$)$.

Comparando os valores da translação crânio-caudal da tíbia entre os grupos com $\mathrm{LCCr}$ rompido e $\mathrm{LCCr}$ corrigido após o procedimento cirúrgico TightRope modificado (tabela 1), observouse que a maioria dos membros operados (12 de 20) apresentou valores de translação crânio-caudal da tíbia $\leq 2,00 \mathrm{~mm}(\mathrm{P}=0,0033)$, sendo o valor médio $1,67 \mathrm{~mm}$. Entretanto, oito animais apresentaram resultados entre 3,00 e 4,00mm após a cirurgia.

Emvirtudedaimportância evulnerabilidade à ruptura, o LCCr é foco de muitos estudos que, em parte, resultaram na descrição de várias técnicas cirúrgicas para correção da RLCCr. O deslocamento cranial da tíbia em relação ao fêmur, denominado movimento de gaveta, é o método diagnóstico utilizado para confirmar a RLCCr (SCHULZ, 2007). Valores superiores a $2,00 \mathrm{~mm}$ sugerem instabilidade do LCCr (MOORE \& READ, 1996).

A modificação da técnica TightRope aqui apresentada contou com a substituição da fita $\left(\right.$ Arthrex $\left.{ }^{\circledR}\right)$ pelo fio de poliamida $(0,7 \mathrm{~mm})$, escolha fundamentada na disponibilidade e baixo custo. Entretanto, em comparação à fita $\left(\right.$ Arthrex $\left.^{\circledR}\right)$, o fio de poliamida tem como desvantagens inferior resistência e maior volume de nó (VIANA \& ROE, 2006; GILES et al., 2008). Neste estudo, o tensionamento do fio de poliamida (implante) em movimentos de extensão e flexão demonstrou resistência e, em dois casos, apresentou rompimentos apenas no aperto final do parafuso ortopédico.

A redução do valor da translação crâniocaudal da tíbia para valores inferiores a $2,00 \mathrm{~mm}$ na maioria dos membros submetidos ao procedimento de TightRope modificado sugere eficácia do método em estabilizar o LCCr, contudo, essa estabilização não foi semelhante à estabilização proporcionada pelo $\mathrm{LCCr}$ intacto, por manter maior movimento de gaveta. $\mathrm{O}$

Tabela 1 - Análise descritiva dos valores da translação crâniocaudal da tíbia previamente à ruptura do ligamento cruzado cranial (LCCr), após a ruptura e após a realização do procedimento cirúrgico TightRope modificado foram $0,50 \mathrm{~mm}, 10,70 \mathrm{~mm}$ e $2,25 \mathrm{~mm}$, respectivamente $(\mathrm{P}=0,0033)$.

\begin{tabular}{lccc}
\hline Dados & LCCr intacto & LCCr rompido & LCCr operado \\
\hline Média & $0.50^{\mathrm{A}}$ & $10.70^{\mathrm{B}}$ & $2.25^{\mathrm{C}}$ \\
Intervalo & 2.00 & 10.00 & 3.00 \\
Mínimo & 0.00 & 6.00 & 1.00 \\
Máximo & 2.00 & 16.00 & 4.00 \\
\hline
\end{tabular}

Ciência Rural, v.43, n.6, jun, 2013. 
custo é definitivamente uma consideração comum e válida na escolha da técnica cirúrgica em medicina veterinária. Como outras técnicas, os custos associados com a cirurgia provavelmente variam amplamente entre os centros. Ao determiná-los, é importante considerar os associados com o tempo anestésico, o uso dos instrumentos caros e esterilização. Dessa forma, é necessária a busca constante por técnicas que simplificam o procedimento, ao mesmo tempo em que possibilitam a redução do valor agregado ao procedimento. Considerando a busca constante por técnicas que estabilizam de forma apropriada a articulação e que cursem com o mínimo de complicações pós operatórias a longo prazo.

Entre as limitações do estudo, está a ausência de mensuração da tensão do nó, que pode, em ciclos repetitivos, interferir na tensão deste e consequente estabilidade da articulação; a padronização da força exercida no teste de gaveta, que, apesar de ser realizada pelo mesmo avaliador, permanece subjetiva; e,. por último, a ausência da avaliação do teste de compressão tibial que poderia ter trazido mais informações a respeito do deslocamento cranial da tíbia durante a avaliação da técnica.

\section{CONCLUSÃO}

A modificação da técnica de TightRope promove estabilidade em joelhos de cadáveres caninos submetidos à ruptura do LCCr. Porém, essa estabilidade é inferior à do ligamento intacto.

\section{COMITÊ DE ÉTICA}

Aprovado pelo Comitê de Ética para uso de Animais (CEA; protocolo no 003-10).

\section{REFERÊNCIAS}

CAROBBI, B.; NESS, M.G. Preliminary study evaluating tests used to diagnose canine cranial cruciate ligament failure. Journal of Small Animal Practice, Gloucester, v.50, p.224-226, 2009.

COMERFORD, E.J. Current thoughts on canine cranial cruciate ligament disease. In: CONGR. INTERNAZ. MUltisala (SCIVAC) 56., 2007, Rimini Proceeding.: Societá culturale italiana veterinari per animali da compangia, 2007. p.147-148.

COMERFORD, E.J. et al. Update on the aetiopathogenesis os canine cranial cruciate ligament disease. Veterinary Comparative Orthopedics and Traumatology, Stuttgart, v.24, n.4, p.91-98, 2011.

CONSTANTINESCU, G.M. Anatomia clínica de pequenos animais. Rio de Janeiro: Guanabara, 2005. p.355.
CONZEMIUS, M.G. et al. Effect of surgical technique on limb function after surgery for rupture of the cranial cruciate ligament in dogs. Journal of the American Veterinary Medical Association, Schaumburg, v.226, n.2, p.232-236, 2005.

COOK, J.L. et al. Clinical comparison os a novel extracapsular stabilization procedur and tibial plateau leveling osteotomy for treatment of cranial cruciate ligament deficiency in dogs. Veterinary Surgery, Philadelphia, v.39, n.3, p.315-323, 2010.

FISCHER, C. et al. Effects of attachment sites and joint angle at the time of lateral suture fixation on tension in the suture for stabilization of the cranial cruciate ligament deficient stifle in dogs. Veterinary Surgery, Philadelphia, v 39. n.10, p.334-342, 2010.

GILES, J.T. et al. Biomerchanical analysis os suture anchors and suture materials in the canine femur. Veterinary Surgery, Philadelphia, v.37, n1 p.12-21, 2008.

GRIFFON, D.J. A review of the pathogenesis of canine cranial cruciate ligament disease as a Basis for future preventive strategies. Veterinary Surgery, Philadelphia, v.39, n.4, p.399409, 2010.

HULSE, D. et al. Determination of isometric points for placement of a lateral suture in treatment of the cranial cruciate ligament deficient stifle. Veterinary Comparative Orthopedics and Traumatology, Stuttgart, v.23, n.4, p.163-167, 2010.

JERRAM, R.M.; WALKER, A.M. Cranial cruciate ligament injury in the dog: pathophysiology, diagnosis and treatment. New Zealand Veterinary Journal, Wellington, v.51, p.149-158, 2003.

KIM, S.E. et al. Tibial osteotomies for cranial cruciate ligament in dogs. Veterinary Surgery, Philadelphia, v.37, n.2, p.111-125, 2008 .

LAZAR, T.P. et al. Long - term radiographic comparison of tibial plateau leveling osteotomy versus extracapsular stabilization for cranial cruciate ligament rupture in the dog. Veterinary Surgery, Philadelphia, v.34, p.133-141, 2005.

LISKA, W.D.; DOYLE, N.D. Canine total knee replacement: surgical technique and one year outcome. Veterinary Surgery, Philadelphia, v.38 n.5, p.568-582, 2009.

MOORE, K.W.; READ, R.A. Rupture of cranial cruciate ligament in dogs-Part II. Diagnosis and management. Compendium on Continuing Education for the Practicing Veterinarian, Yardley, PA, v.18, n.4, p.381-391, 1996.

PALMER, R.H. Diagnosing cranial cruciate ligament pathology. Veterinary Medicine, Lenexa, v.41, n.7, p.414-422, 2005.

PIERMATTEI, D.L. et al. The Stifle joint. In: Handbook of small animal - orthopedics and fracture repair. 4.ed. Philadelphia: Saundres, 2006. p.818.

PIERMATTEI, D.L.; GREELEY, R.G. Atlas de abordagens cirúrgicas aos ossos do cão e gato. São Paulo: Manole, 2004. p.197.

ROOSTER, H.D. et al. Morphologic and functional fratures of the canine cruciate ligaments. Veterinary Surgery, Philadelphia, v.8, n.35, p.769-780, 2006.

Ciência Rural, v.43, n.6, jun, 2013. 
SCHULZ, K.; STIFLE. Cranial cruciate rupture ligament rupture. In: FOSSUM, T.W. Manual of small animal surgery. 3.ed. St. Louis: Mosby, 2007. p.1254-1275.

TONKS C.A, et al. The effects of extra-articular suture tension on contact mechanics of the lateral compartment of cadaveric stifles treated with the TightRope CCL or lateral suture technique. Veterinary Surgery, Philadelphia, v.39, n.3, p.399-409, 2010.
VASSEUR, P.B. Stifle joint. In: SLATTER, D. Textbook of small animal surgery. 3.ed. Philadelphia: Saunders, 2003. V.II, Chapt.147, p.2090-2116.

VIANNA, M.L.; ROE, S.C. Mechanical comparison of two knots and two crimp systems for securing nylon line used for extraarticular stabilization of the canine stifle. Veterinary Surgery, Philadelphia, v.35, n.6, p.567-572, 2006.

Ciência Rural, v.43, n.6, jun, 2013. 